\title{
Diagnostic Confidence and Prognosis in Fibrotic Hypersensitivity Pneumonitis: Classification Is Fundamental
}

\author{
Vasilios Tzilas $^{a}$ Demosthenes Bouros ${ }^{a, b}$ \\ ${ }^{a}$ Center of Excellence for Diseases of the Chest, Athens Medical Center, Athens, Greece; ${ }^{b}$ Center of Excellence for \\ Diseases of the Chest, Athens Medical Center, Medical School, National and Kapodistrian University of Athens, \\ Athens, Greece
}

The field of interstitial lung diseases is especially challenging in terms of diagnosis. The backbone of the diagnostic procedure is multidisciplinary discussion. Unfortunately, multidisciplinary discussion is not a diagnostic panacea. Hypersensitivity pneumonitis (HP) represents a characteristic example where diagnostic agreement is disappointing even among experienced multidisciplinary teams $(\kappa w=0.29)$ [1]. Quite often it is not possible to achieve a definite diagnosis, and for practical reasons, the physicians proceed to management decisions based on a working or provisional diagnosis. Ryerson et al. [2] offered a standardized ontological framework for fibrotic ILD based on the degree of diagnostic confidence. This served as a basis for diagnostic algorithms [3,4] and eventually the first diagnostic guideline for HP [5]. It is firmly established that fibrotic HP (f-HP) has a worst prognosis comparing to nonfibrotic HP. Nevertheless, the former still represents a heterogeneous group with variable course and outcome. Studies exploring prognostic parameters in $\mathrm{f}-\mathrm{HP}$ are important as they will provide valuable information regarding management decisions.

In this issue of Respiration, Kang et al. [6] studied retrospectively the prognostic implication of diagnostic confidence level in 101 patients with biopsy-proven f-HP.
The patients were classified according to the diagnostic algorithm proposed by Vasakova et al. [4]. This classification schema is based on the degree of diagnostic confidence following evaluation of exposure to an inciting antigen (history and serum-specific IgGs), bronchoalveolar lavage fluid (BALF) cellular analysis, imaging (high-resolution computed tomography), and pathology findings. It includes 4 categories, confident, probable, possible, and unlikely HP. The majority of patients were classified as possible HP (51.5\%), followed by probable (26.7\%), confident $(9.9 \%)$, and unlikely (6.9\%) HP while 5 patients could not be classified. The main finding from this study is that there were distinctive survival curves according to the degree of diagnostic confidence. Higher diagnostic confidence was correlated with improved survival. Specifically, patients with unlikely HP exhibited a significantly increased mortality comparing to patients with probable ( $p=0.009)$ and confident $(p=0.03) \mathrm{HP}$.

In unadjusted Cox analysis, other factors significantly associated with increased mortality were older age, lower lung function (FVC and DLco), lower 6-min walk distance, and lower nadir saturation during the 6-min walk test. These findings could be attributed to lead-time bias. Another interesting finding is that lower BALF lympho- karger@karger.com www.karger.com/res

(c) 2021 S. Karger AG, Base

Karger"
Correspondence to:

Vasilios Tzilas, tzilasvasilios@gmail.com Demosthenes Bouros, dbouros@med.uoa.gr 
cytosis was also significantly correlated with increased mortality. It is not known whether patients with $\mathrm{f}-\mathrm{HP}$ and reduced BALF lymphocytosis represent a distinct phenotype with worst prognosis or if BALF lymphocytosis decreases in the course of time and thus reduced BALF lymphocytosis is also subject to lead-time bias. Since BALF lymphocytosis was strongly correlated with the confidence level in HP diagnosis, the authors performed 2 Cox multivariable models to evaluate the significance of prognosticators. In multivariable analysis, unlikely HP remained an independent predictor of mortality.

It should be noted that the category of unlikely HP is characterized by absence of imaging findings characteristic of airway involvement (e.g., the 3-density pattern or ill-defined centrilobular nodules), negative history of exposure, and lack of BALF lymphocytosis. In such cases and in the absence of typical histological features of $\mathrm{f}-\mathrm{HP}$ (i.e., chronic fibrosing interstitial pneumonia or airwaycentered fibrosis and the presence of poorly formed nonnecrotizing granulomas), one should reassess the diagnosis of HP and consider alternative diagnoses that could be related to a worst prognosis. A characteristic example is antisynthetase syndrome that can masquerade as HP. We recently described 4 cases that were initially diagnosed as f-HP. All patients reported exposure to an inciting antigen that inevitably strongly raised the suspicion of HP. Furthermore, 2 patients had pronounced BALF lymphocytosis ( $\geq 30 \%$ ), while 2 had lung biopsy findings suggestive of HP (probable HP). No patient reported muscle weakness, and serology testing was negative in all patients. Final diagnosis was achieved by ordering a dedicated myositis panel. In these cases, even the histological information provided by a surgical lung biopsy was unable to establish the correct diagnosis. In patients with nonspecific interstitial pneumonia/organizing pneumonia imaging pattern, even in the absence of extrapulmonary symptoms and signs, it is important to meticulously exclude the possibility of antisynthetase syndrome [7].

The longitudinal course of FVC is an established marker of disease progression and mortality in IPF and fibrotic ILDs. Thus, as expected, longitudinal change of FVC was also correlated with the degree of diagnostic confidence. The confident HP group actually showed improvement in FVC (1.0\% pred/month) that was significantly different from the possible $(-0.5 \%$ pred/month; $p=0.001)$ and unlikely $(-0.6 \% \mathrm{pred} / \mathrm{month} ; p=0.038)$ HP group.

Another seemingly minor but important point is the moderate agreement $(\kappa=0.575)$ that was achieved between thoracic radiologists in determining HRCT pat- terns. HRCT interpretation is an extremely vital area as it plays a pivotal role in the diagnostic investigation of ILDs. The difficulties and disagreement in HRCT interpretation is a known fact, even between experienced thoracic radiologists [8]. In everyday practice, a dedicated thoracic radiologist is not always available, which further intensifies the problem [9].

The main limitations of this study are its retrospective and unicentric nature and the relatively small number of patients. Of note, the unlikely HP group included only 7 patients which weaken the results of the statistical analysis. Larger multicentric studies are required. The participation of many centers will increase the homogeneity at a diagnostic level as well as the validity of the statistical analysis.

The first clinical practice guidelines for HP by ATS/ JRS/ALAT [5] represent a significant first step toward universal diagnostic harmonization [10]. It would be useful for the next studies exploring the prognostic value of diagnostic confidence to be based on these guidelines. Furthermore, it is important to keep in mind that the introduction of the classification of idiopathic interstitial pneumonias according to disease behavior [11] revolutionized the way we approach them from a diagnostic and management perspective. This concept of classification according to longitudinal disease behavior has transcended the boundaries of IIPs and was found to be universally applicable to other fibrotic ILDs as well. It ultimately led to the establishment of the progressive fibrotic phenotype as a key element that can help therapeutic decisions. The definition of progressive fibrotic phenotype is based on 3 pillars, clinical, physiological, and imaging. We expect that the upcoming IPF guidelines will address this issue, providing more clear guidance on how to define this phenotype and promote its application and validation in the field of fibrotic ILDs including f-HP.

\section{Conflict of Interest Statement}

The authors have no conflicts of interest to declare.

\section{Funding Sources}

No funding was received.

\section{Author Contributions}

V.T. and D.B. both wrote and reviewed the manuscript. 


\section{References}

1 Walsh SLF, Wells AU, Desai SR, Poletti V, Piciucchi S, Dubini A, et al. Multicentre evaluation of multidisciplinary team meeting agreement on diagnosis in diffuse parenchymal lung disease: a case-cohort study. Lancet Respir Med. 2016 Jul;4(7):557-65.

2 Ryerson CJ, Corte TJ, Lee JS, Richeldi L, Walsh SLF, Myers JL, et al. A standardized diagnostic ontology for fibrotic interstitial lung disease. An International Working Group perspective. Am J Respir Crit Care Med. 2017 Nov 15;196(10):1249-54.

3 Salisbury ML, Myers JL, Belloli EA, Kazerooni EA, Martinez FJ, Flaherty KR. Diagnosis and treatment of fibrotic hypersensitivity pneumonia. Where we stand and where we need to go. Am J Respir Crit Care Med. 2017 Sep 15; 196(6):690-9.
4 Vasakova M, Morell F, Walsh S, Leslie K, Raghu G. Hypersensitivity pneumonitis: perspectives in diagnosis and management. Am J Respir Crit Care Med. 2017 Sep 15;196(6): 680-9.

5 Raghu G, Document S, Remy-Jardin M, Ryerson CJ, Myers JL, Kreuter M, et al. Diagnosis of hypersensitivity pneumonitis in adults: an official ATS/JRS/ALAT clinical practice guideline. Am J Respir Crit Care Med. 2021 Jan 1;203(1):150-1.

6 Kang J, Kim YJ, Choe J, Chae EJ, Song JS, Song JW. Prognostic implication of diagnostic confidence level in patients with fibrotic hypersensitivity pneumonitis. Respiration. 2021.

7 Tzilas V, Sfikakis PP, Bouros D. Antisynthetase syndrome masquerading as hypersensitivity pneumonitis. Respiration. 2021 Jun 18: $1-9$.
8 Walsh SL, Calandriello L, Sverzellati N, Wells AU, Hansell DM. Interobserver agreement for the ATS/ERS/JRS/ALAT criteria for a UIP pattern on CT. Thorax. 2016 Jan;71(1):45-51.

9 Tzilas V, Tzouvelekis A, Chrysikos S, Papiris S, Bouros D. Diagnosis of idiopathic pulmonary fibrosis "pragmatic challenges in clinical practice”. Front Med. 2017;4:151.

10 Tzilas V, Tzouvelekis A, Bouros D. Hypersensitivity pneumonitis: the first diagnostic guidelines. Lancet Respir Med. 2020 Oct; 8(10):955-7.

11 Travis WD, Costabel U, Hansell DM, King TE Jr, Lynch DA, Nicholson AG, et al. An official American Thoracic Society/European Respiratory Society statement: update of the international multidisciplinary classification of the idiopathic interstitial pneumonias. Am J Respir Crit Care Med. 2013 Sep 15;188(6):733-48. 